\title{
Padrões temporais do vento à superfície em mesorregiões do estado da Bahia
}

\author{
Temporal pattern of wind surface in mesorregions of the Bahia State
}

\author{
Washington Luiz Félix Correia Filho*1, Maria Regina da Silva Aragão² \\ ${ }^{1}$ Programa de Pós-Graduação em Ciências Climáticas, Universidade Federal do Rio Grande do Norte, Natal, \\ Brasil \\ ${ }^{2}$ Universidade Federal de Campina Grande, Campina Grande, Brasil
}

\begin{abstract}
Resumo
O objetivo deste trabalho foi determinar padrões de variabilidade temporal do vento à superfície em mesorregiões do estado da Bahia. Foram utilizados métodos de análise multivariada (análise de componentes principais e análise de agrupamento) aplicados a médias trimestrais das componentes do vento à superfície registrado por cinco plataformas automáticas de coleta de dados operadas pelo Instituto do Meio Ambiente e Recursos Hídricos (INEMA). Os anos estudados foram 2000, 2002, 2005, 2006 e 2007. As duas primeiras componentes principais da componente zonal do vento explicaram $99,4 \%$ da variância total das médias trimestrais, enquanto que as duas primeiras da componente meridional explicaram $99,6 \%$. A análise de agrupamento aplicada aos fatores comuns temporais mais significativos através do método de Ward e distância euclidiana resultou em três grupos homogêneos para cada componente do vento, dos quais dois estão constituídos por uma estação apenas. Esses grupos se caracterizam por velocidades do vento mais elevadas, um resultado que sugere a importância de fatores locais como o aspecto do relevo.
\end{abstract}

Palavras-chave: vento à superfície, análise multivariada, regionalização, Bahia, Nordeste do Brasil.

\begin{abstract}
The objective in this study was to determine the temporal variability patterns of the surface wind in mesoregions of the Bahia state. Multivariate analysis methods (principal component analysis and cluster analysis) were applied to three-month averages of the zonal and meridional components of the surface wind registered by five automatic stations operated by the Instituto do Meio Ambiente e Recursos Hídricos (INEMA). The years studied were 2000, 2002, 2005, 2006 and 2007. The first two principal components of the zonal wind component explained 99.4\% of the total variance of the three-month averages, while the first two principal components of the meridional wind component explained $99.6 \%$. The cluster analysis applied to the most significant temporal factor loadings by means of the Ward method and Euclidian distance resulted in three homogeneous groups for each wind component, with two groups being comprised by one station only. Higher wind speeds characterize these groups, a result that points toward the importance of local factors such as the aspect of the relief.
\end{abstract}

Keywords: surface wind, multivariate analysis, regionalization, Bahia, Northeast Brazil.

*wlfcfm@gmail.com

Recebido: 14/03/2014 Revisado: 19/05/2014 Aceito: 29/04/2014 


\section{Introdução}

A discussão político-científica sobre as mudanças climáticas globais mostra a necessidade de ampliar o conhecimento sobre sistemas climáticos e, conseqüentemente, sobre a atmosfera e sua interação com a superfície terrestre. Nesse contexto o vento, responsável pelo transporte horizontal de propriedades na atmosfera, é uma variável cuja importância pode ser avaliada pela diversidade de trabalhos nos quais é analisado, seja como foco principal da investigação, seja como elo fundamental no entendimento de fenômenos atmosféricos, particularmente os que resultam da interação superfície-atmosfera.

$\mathrm{O}$ vento tem influência significativa em diversas áreas tais como saúde (QIAN et al.,2007; BARMPADIMOS et al., 2011), geração de energia (SILVA et al., 2002; ARCHER \& JACOBSON, 2005), etc. Na saúde está diretamente ligado à qualidade do ar e de vida da população, através da dispersão e transporte de poluentes comuns nas grandes cidades (HUANG et al., 2009; AIKAWA et al., 2008). No setor energético brasileiro, sua importância estratégica tem aumentado nos últimos anos com a implementação da política de novas fontes alternativas, visando produzir energia de maneira sustentável, sem degradar o meio ambiente (SILVA et al., 2002; MUÑOZ \& GÁNDARA, 2002). Independentemente do objetivo, é importante caracterizar os regimes de vento através da determinação de padrões de circulação em diversas escalas de tempo e espaço. Assim, diversas pesquisas têm sido realizadas com o objetivo de identificar fatores que contribuem para a variabilidade do vento e/ou sua interação com outras variáveis meteorológicas, principalmente a precipitação, a exemplo de Moscati \& Gan (2007), que investigaram a variabilidade da precipitação no período chuvoso da região semi-árida do Nordeste do Brasil e sua relação com o regime de vento. Eles concluíram que os totais pluviométricos são menores quando as componentes do vento (leste e sul) estão mais fortes sobre a região, e que os totais pluviométricos são maiores quando elas estão enfraquecidas.

Diversos métodos estatísticos têm sido utilizados para caracterizar os regimes do vento. Um dos mais difundidos é a análise multivariada que tem sido aplicada ao vento com o objetivo de: caracterizar condições climatológicas (GREEN et al.,1993; KLINK, 1999; KAUFMANN \& WHITEMAN, 1999), identificar regimes climáticos e regiões homogêneas (WEBER \& FURGER, 2001; BURLANDO et al., 2008; JIMENEZ et al., 2008), identificar tipos de circulação atmosférica (KASSOMENOS et al., 2003), e identificar relações com outras variáveis meteorológicas (CHAVES \& CAVALCANTI, 2001). Técnicas de análise multivariada também tem possibilitado a identificação de regiões homogêneas e de regimes climáticos do vento à superfície no Nordeste do Brasil (CORREIA, 2000; BARRETO, 2001; CORREIA FILHO, 2010; OLIVEIRA SILVA et al., 2011). Neste trabalho elas são utilizadas para caracterizar padrões de variabilidade temporal do vento à superfície em mesorregiões da Bahia.

\section{Materiais e métodos}

\section{1 Área de estudo}

A região de estudo é a Bahia, o maior estado do Nordeste do Brasil, cuja área de $564.692 \mathrm{~km}^{2}$ corresponde a $36,41 \%$ da área total da região. Seu relevo é constituído por planícies, vales, serras e montanhas com altitude entre $800 \mathrm{~m}$ e $1200 \mathrm{~m}$, como na Chapada Diamantina (Figura 1).

A Chapada Diamantina, a maior estrutura orográfica da Bahia, afeta a circulação atmosférica contribuindo para que a vertente leste seja mais úmida que a vertente oeste que apresenta características do semi-árido (BRAGA et al., 1998).

O tempo e clima da Bahia resultam da interação entre a superfície e mecanismos atmosféricos de escala global, regional e local. O anticiclone subtropical do Atlântico sul (ASAS) domina a circulação atmosférica no estado, em média. Diversos sistemas de tempo também influenciam o território baiano tais como: Zona de Convergência Intertropical (ZCIT) no extremo norte do estado, Vórtice Ciclônico de Ar Superior (VCAS), Zona de Convergência do Atlântico Sul (ZCAS) no sul do estado, sistemas frontais, ondas/distúrbios de leste, sistemas convectivos de mesoescala, brisas marítima/ terrestre e ventos de vale/montanha (KOUSKY, 1979; MOLION \& BERNARDO, 2002; CHAVES \& CAVALCANTI, 2001; SANTOS, 2008; OLIVEIRA SILVA et al., 2009; SANTOS et al., 2012).

\subsection{Dados}

Os dados utilizados neste trabalho foram obtidos dos registros de cinco Plataformas Automáticas de Coletas de Dados (PCD's) operadas pelo Instituto do Meio Ambiente e Recursos Hídricos (INEMA) nas seguintes mesorregiões da Bahia: Mesorregião Nordeste Baiano - Serrinha, Mesorregião Metropolitana de Salvador Salvador, Mesorregião do Sudeste Baiano - Ilhéus e Mesorregião Sul Baiano - Eunápolis e Teixeira de Freitas. Instaladas no final da década de 90 as PCD's, cuja localização é ilustrada na Figura 2, armazenam valores de diversas variáveis meteorológicas além da direção e da velocidade do vento.

Foi necessário processar os arquivos originais (dados brutos) das PCD's de forma a extrair os dados necessários à pesquisa. Concluído o processamento, todo o conjunto de dados foi subjetivamente avaliado com o objetivo de identificar os períodos de observação comuns as cinco PCD's, o que resultou na seleção dos anos de 2000, 2002, 2005, 2006 e 2007. O Quadro 1 contém o número total de arquivos e as coordenadas geográficas de cada estação. 


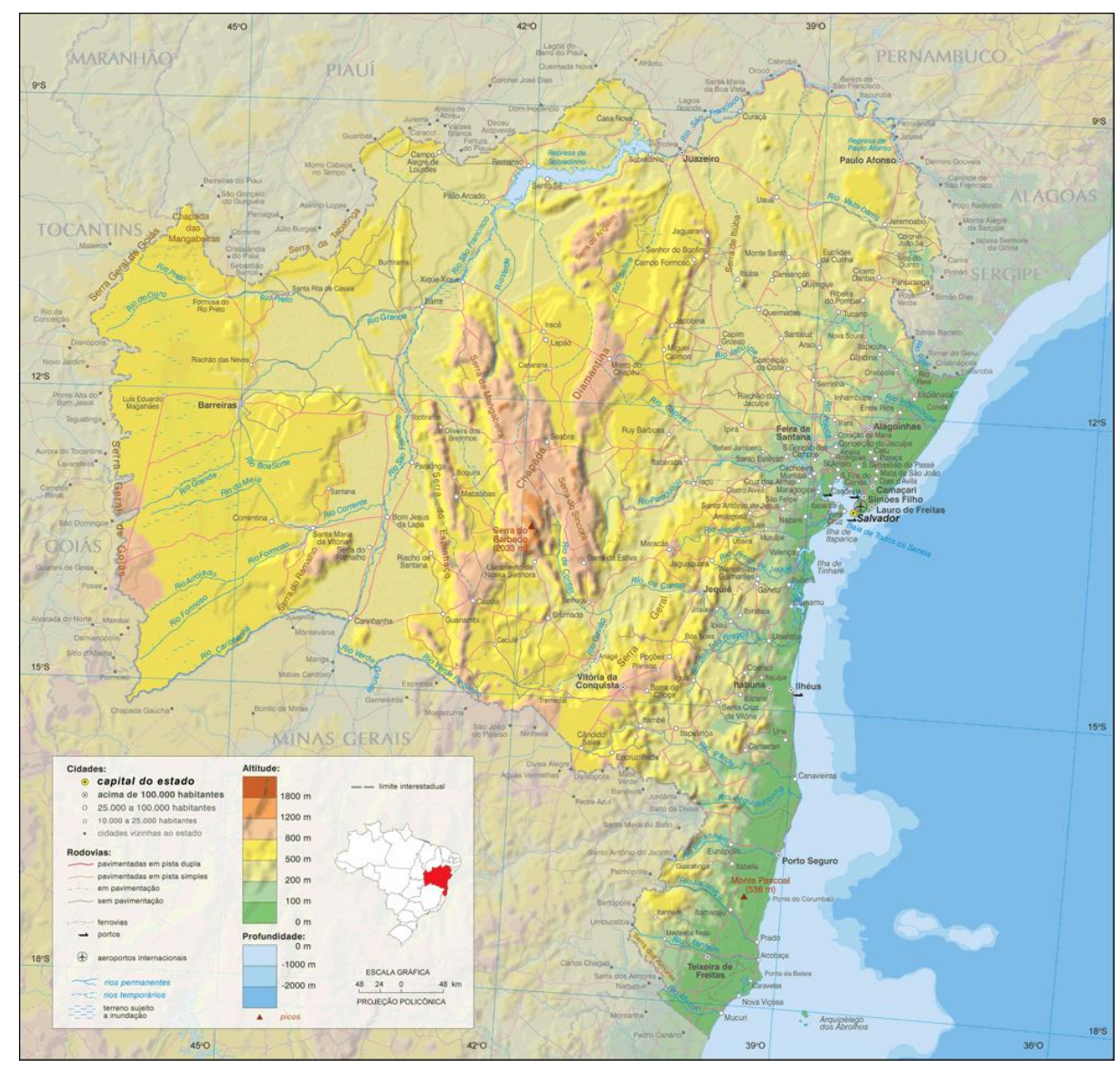

Figura 1 - Mapa do relevo do Estado da Bahia. A legenda de cores representa a altitude com a seguinte correspondência: verde escuro, entre 0 e 200m; amarelo, entre 200-800m; magenta, entre 800-1200m; e laranja, entre 1200-1800m (Fonte: IBGE, 2012).

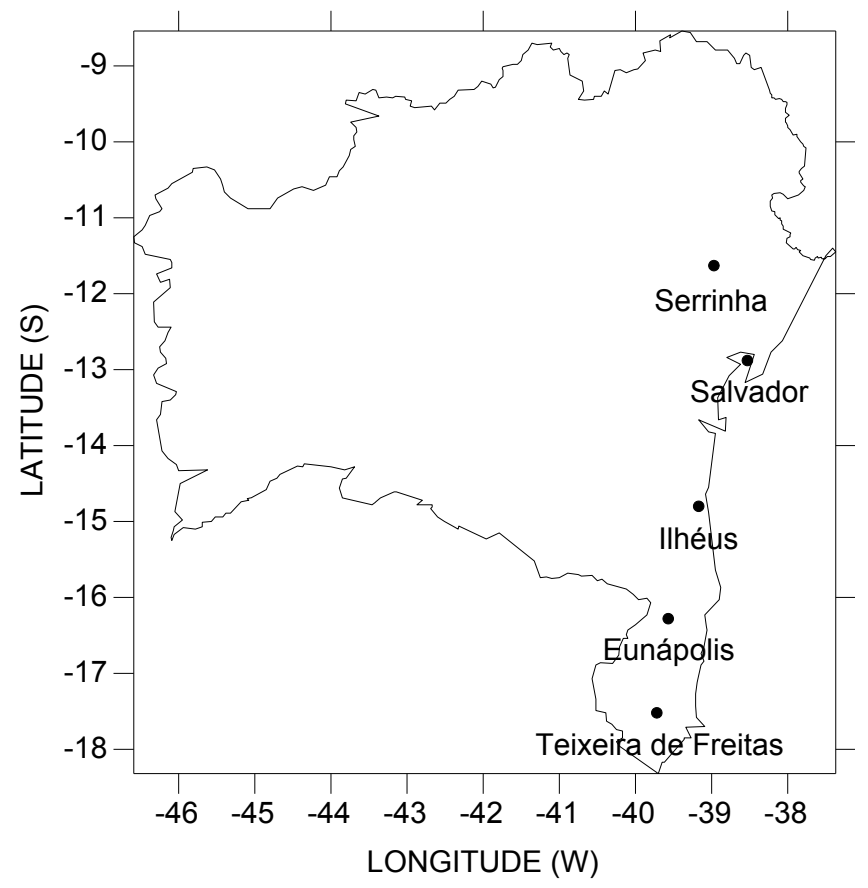

Figura 2 - Localização das Plataformas Automáticas de Coletas de Dados (PCD's) em mesorregiões da Bahia. 
Quadro 1 - Nome, coordenadas geográficas e número de arquivos de dados das Plataformas Automáticas de Coleta de Dados (PCD's) em mesorregiões do estado da Bahia.

\begin{tabular}{l|c|c|c|c}
\hline \multicolumn{1}{c|}{ Localidade } & $\begin{array}{c}\text { Latitude } \\
\mathbf{( S )}\end{array}$ & $\begin{array}{c}\text { Longitude } \\
\mathbf{( W )}\end{array}$ & $\begin{array}{c}\text { Altitude } \\
\mathbf{( m )}\end{array}$ & $\begin{array}{c}\text { Número de } \\
\text { Arquivos }\end{array}$ \\
\hline Serrinha & $11^{\circ} 38^{\prime} 54^{\prime \prime}$ & $38^{\circ} 58^{\prime} 58^{\prime \prime}$ & 336 & 20 \\
\hline Salvador & $12^{\circ} 55^{\prime} 53^{\prime \prime}$ & $38^{\circ} 21^{\prime} 40^{\prime \prime}$ & 25 & 64 \\
\hline Ilhéus & $14^{\circ} 48^{\prime} 00^{\prime \prime}$ & $39^{\circ} 10^{\prime} 48^{\prime \prime}$ & 62 & 18 \\
\hline Eunápolis & $16^{\circ} 17^{\prime} 26^{\prime \prime}$ & $39^{\circ} 34^{\prime} 47^{\prime \prime}$ & 179 & 20 \\
\hline Teixeira de Freitas & $17^{\circ} 31^{\prime} 48^{\prime \prime}$ & $39^{\circ} 43^{\prime} 12^{\prime \prime}$ & 195 & 10 \\
\hline
\end{tabular}

\subsection{Métodos}

O vento à superfície dos registros de cada estação automática foi decomposto em suas componentes zonal (u) e meridional (v) usando, respectivamente, as equações:

$$
\begin{aligned}
& u=-V \cdot \operatorname{sen} \theta \\
& v=-V \cdot \cos \theta
\end{aligned}
$$

em que $\mathrm{V}$ é a velocidade do vento $\left(\mathrm{ms}^{-1}\right)$ e $\theta$ é a direção do vento em radianos.

Neste estudo, médias trimestrais para o conjunto dos cinco anos foram submetidas a técnicas multivariadas, em separado para cada componente: análise de componentes principais (ACP) e análise de agrupamento (AA). Inicialmente, foi necessário calcular as médias mensais para cada ano selecionado. Dois critérios foram utilizados para selecionar os meses cuja média mensal seria utilizada. O primeiro está relacionado com o número de observações horárias existente em cada dia do mês, e determina que apenas as médias dos dias com, no mínimo, 12 observações horárias fossem incluídas no cálculo da média mensal. O segundo critério está relacionado com o número de médias diárias existente no mês, e determina que apenas os meses com, no mínimo, 15 médias diárias tivessem a sua média mensal incluída no cálculo da média mensal do conjunto dos anos analisados. Obedecendo a esses critérios, foi possível utilizar as médias de todos os meses do período de estudo.

\subsubsection{Análise de Componentes Principais (ACP)}

Esta técnica de análise multivariada é amplamente utilizada em Meteorologia. Ela possibilita a redução do número de variáveis de um conjunto de dados, preservando a sua variância total, e a identificação de padrões e processos associados às variáveis (WILKS,
2005; CORRAR et al., 2007). Neste trabalho a matriz $F(P x N)$ é constituída de $P$ estações, e $\mathrm{N}$ observações (médias trimestrais da componente zonal ou meridional) em cada uma das estações. Dessa forma,

$$
F=\left[\begin{array}{cccc}
f_{11} & f_{12} & \cdots & f_{1 n} \\
f_{21} & f_{22} & \cdots & f_{2 n} \\
\vdots & \vdots & \cdots & \vdots \\
f_{p 1} & f_{p 2} & \cdots & f_{p n}
\end{array}\right]
$$

Cada linha da matriz $F$ representa uma estação $P$ e as colunas representam as observações na estação $P$. A matriz de correlação $R$ é obtida a partir da matriz $F$ das variáveis padronizadas pelos desvios padrões, dada por:

$$
R=\frac{1}{N-1} F^{T} F
$$

$R$ uma matriz simétrica positiva de dimensão $(P x$ $P$ ) e diagonalizável por uma matriz $E$, denominada de autovetores. A matriz diagonal $D$, cujos elementos diagonais são os autovalores de $R$ é obtida por:

$$
D=E^{-1} R E
$$

Em virtude da ortogonalidade dos autovalores, a matriz inversa de $E=\left(E^{-1}\right)$ é igual a sua transporta $\left(E^{T}\right)$. Assim as CP's $U_{1}, U_{2}, \cdots, U_{b}$ são obtidas pela combinação linear entre a matriz dos autovetores de $\left(E^{T}\right)$ e a matriz de observações $F$, ou seja,

$$
U=E^{T} F \quad \text { ou } \quad F=E U
$$

Cada linha de $U$ é uma $C P$ que são séries temporais associadas aos vetores. Cada CP $U_{1}, U_{2}, \cdots, U_{p}$ tem uma porção da variância total dos dados em ordem decrescente dos autovetores mais significativos $e_{p}$ em E. Logo, 


$$
U=\sum_{i=1}^{p} e_{i p} F_{p}
$$

Em que $F_{p}$ é a matriz original normalizada e $e_{p}$ é o autovetor. Os valores de $\mathrm{F}$ do n-ésimo local podem ser estimados pela seguinte expressão:

$$
F_{p}=e j_{1} U_{1}+e j_{2} U_{2}+e j_{3} U_{3}+\cdots+a_{n b} U_{p}
$$

Todas as combinações possíveis de três meses consecutivos foram analisadas para verificar se a matriz de dados era adequada para a aplicação da ACP. O valor do determinante e do teste Kaiser-Meyer-Olkin (KMO) (CORRAR et al., 2007) foram considerados em todos os casos. Dessa forma, a matriz formada pelas médias de fevereiro-março-abril (FMA), maio-junho-julho (MJJ), agosto-setembro-outubro (ASO) e novembro-dezembrojaneiro (NDJ) foi selecionada por apresentar os melhores resultados.

\subsubsection{Análise de Agrupamento (AA)}

Esta técnica tem por objetivo propor uma estrutura classificatória ou de reconhecimento da existência de grupos, ou seja, a partir de um conjunto de n indivíduos medidos em $p$ variáveis obter um esquema que possibilite reunir indivíduos num certo número de grupos, tal que exista homogeneidade dentro dos grupos e heterogeneidade entre os grupos. Na Meteorologia esta técnica tem sido usada para identificar regiões climatologicamente homogêneas (regionalização), quer sejam obtidas de um grupo de estações ou de período de tempo (dia, mês, ano, etc.), extraindo o comportamento padrão dos eventos (GONG \& RICHMANN, 1995). No processo de extração dos grupos são necessários dois passos: (a) definir uma medida de distância $\mathrm{d}$ a fim de estabelecer similaridades entre os pares de elementos, e (b) definir a função de agrupamento a ser utilizada no procedimento (EVERITT, 1993).

Dentre os diversos tipos de medidas de distância, foi usada a distância Euclidiana obtida por:

$$
d_{a b}=\left[\frac{1}{N_{a b}} \sum_{j=1}^{N_{a b}}\left(X_{a j}-X_{b j}\right)^{2}\right]^{1 / 2}
$$

em que:

$X_{a j}$ é a j-ésima característica da a-ésima observação;

$X_{b j}$ é a j-ésima característica da b-ésima observação;

$N_{a b}$ é o número de estações.
Assim como para a medida de distância, há vários métodos de agrupamento. Neste trabalho foi utilizado o método hierárquico de Ward (KALKSTEIN, 1987; WEBER \& FURGER, 2001; BURLANDO et al., 2008). Essa técnica, desenvolvida por Ward (1963), se baseia na soma dos desvios quadráticos em relação à média do grupo para identificar similaridade, expressa por:

$$
W=\sum_{k=1}^{K} \sum_{j=1}^{J} \sum_{i=1}^{N_{k}}\left(X_{i j k}-X_{\bullet j k}\right)^{2}
$$

em que $X_{i j k}$ é o valor da j-ésima variável para a i-ésima das $N_{k}$ observações dos K grupos e $X_{\bullet j k}$ é o valor médio.

O método de Ward busca minimizar a soma quadrática dos desvios dentro de cada grupo, pois a distância quadrática entre os grupos é essencial na aplicação da técnica. Outro aspecto importante nesse método é o número de observações $N_{k}$; se as distâncias entre uma observação e dois grupos são idênticas, a observação se unirá ao grupo que tiver menos observações.

\section{Resultados e discussões}

\subsection{Análise de componentes principais}

As correlações temporais trimestrais das duas primeiras componentes principais da componente zonal do vento à superfície (CZV), que explicam $99,4 \%$ da variância total, são ilustradas na Figura 3. O primeiro fator comum temporal, que explica $96,1 \%$ da variância, exibe correlações entre 0,51 e 0,86 , enquanto que o segundo fator comum temporal, que explica $3,3 \%$ da variância total, tem correlações entre 0,51 e 0,86 . Observando as correlações para os dois fatores comuns temporais, constata-se que para o primeiro fator comum temporal as maiores correlações estão nos trimestres NDJ e FMA, enquanto que para o segundo fator elas estão em MJJ e ASO, o que sugere influência da ZCAS e do VCAS/ VCAN (trimestres NDJ e FMA), e dos distúrbios de leste (trimestres MJJ e ASO). Além desses sistemas meteorológicos, há também a contribuição do ASAS e dos sistemas frontais durante todo o ano.

Os padrões de variabilidade espacial das duas primeiras componentes principais $(\mathrm{CP})$ da $\mathrm{CZV}$ são mostrados na Figura 4. A primeira CP (Figura 4a) associada a este fator mostra contribuições positivas em Eunápolis e Ilhéus, com valor máximo de 1,34 em Ilhéus. As contribuições negativas são registradas em Salvador, Teixeira de Freitas e Serrinha, com valor mínimo de $-1,24$ na última. Esse padrão de contribuições positivas e negativas indica que há contraste entre as estações com contribuições de sinais opostos devido a sistemas atmosféricos como o VCAS/VCAN (estações localizadas na parte norte) e 


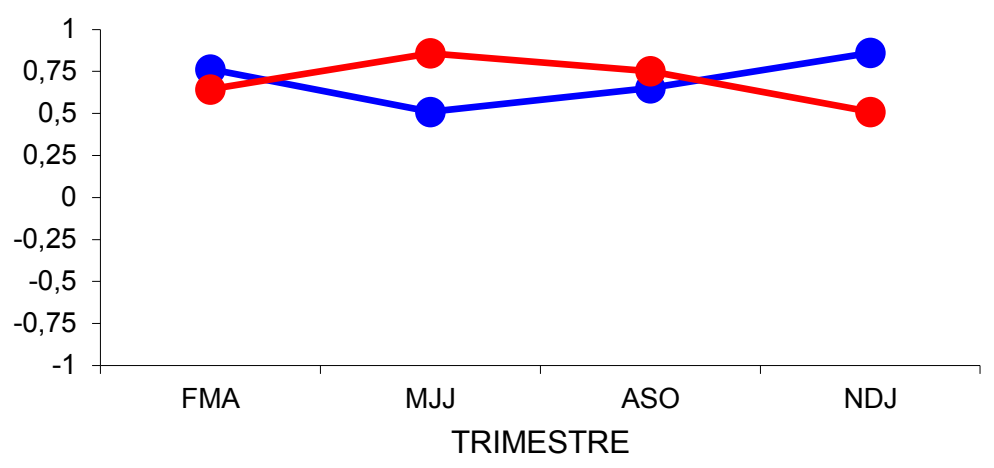

Figura 3 - Correlações do primeiro (azul) e segundo (vermelho) fatores comuns temporais da componente zonal do vento à superfície em mesorregiões do estado da Bahia. (Fonte dos dados: Instituto do Meio Ambiente e Recursos Hídricos (INEMA))
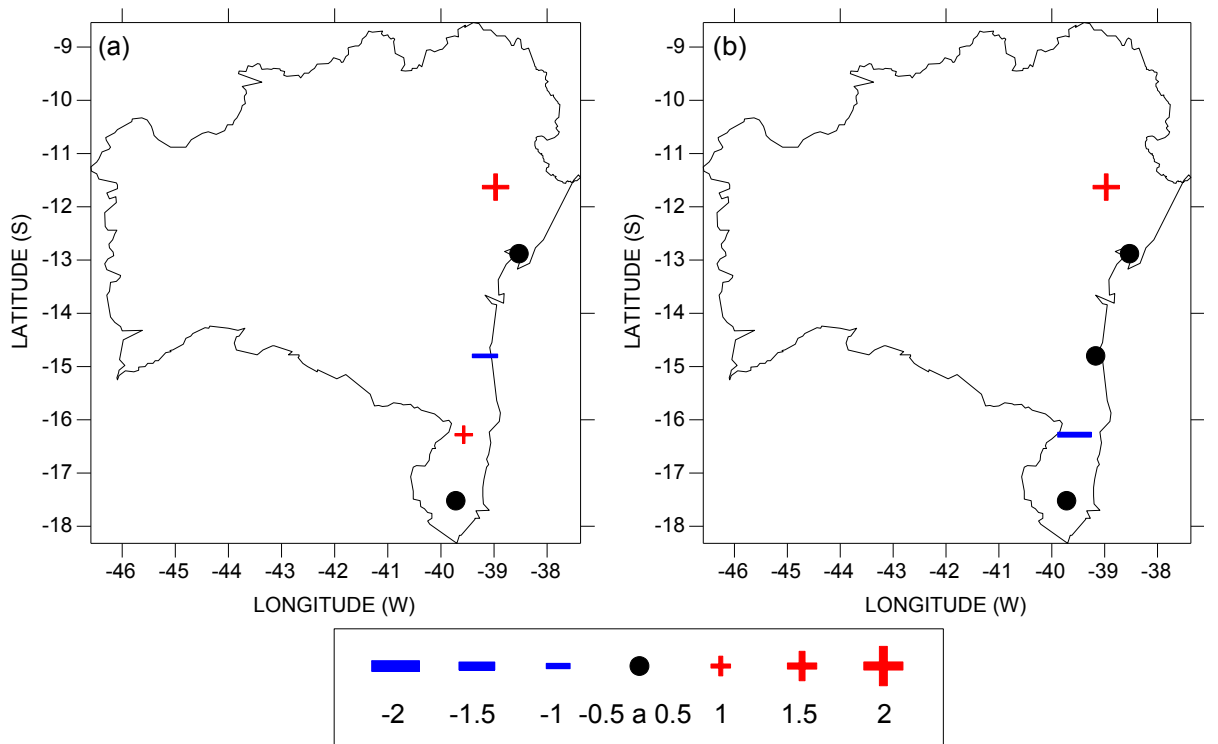

Figura 4 - Padrões de variabilidade espacial do primeiro (a) e segundo (b) fatores comuns temporais da componente zonal do vento à superfície em mesorregiões do estado da Bahia. As contribuições positivas estão representadas pelo símbolo (+) e as negativas pelo símbolo (-). (Fonte dos dados: Instituto do Meio Ambiente e Recursos Hídricos (INEMA))

ZCAS/sistemas frontais (estações localizadas na parte sul).

A segunda CP (Figura $4 \mathrm{~b}$ ) associada a este fator mostra contribuições positivas em Teixeira de Freitas, Ilhéus e Salvador, com valor máximo de 1,17 na última, enquanto que em Serrinha e Eunápolis as contribuições são negativas, com valor mínimo de $-1,34$ na última. Esta componente sugere a resposta local a sistemas atmosféricos como o ASAS, os distúrbios de leste e os sistemas frontais.

As correlações temporais trimestrais das duas primeiras componentes principais da componente meridional do vento à superfície (CMV), que explicam 99,6\% da variância total, são ilustradas na Figura 5.

O primeiro fator comum temporal, que explica $92,4 \%$ da variância total, exibe correlações entre 0,41 e 0,92, enquanto que o segundo fator comum temporal, que explica 7,2\% da variância total, tem correlações entre 0,38 e 0,91 . A partir das correlações dos dois fatores comuns temporais, constata-se que a componente meridional exibe variação sazonal semelhante a da componente zonal, porém a componente principal com correlação mais alta em cada trimestre difere entre as duas componentes do vento. Assim, as correlações mais altas do primeiro fator comum temporal estão nos trimestres MJJ e ASO, enquanto que para o segundo fator comum temporal o valor mais alto está em NDJ. Os dois fatores comuns temporais tem correlações iguais em FMA.

Os padrões de variabilidade espacial das duas primeiras componentes principais (CP) da componente meridional do vento são mostrados na Figura 6. A 


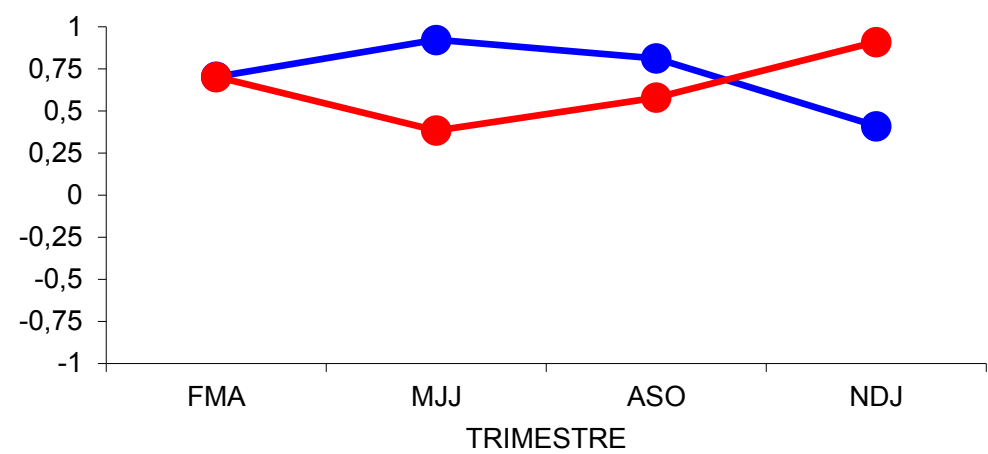

Figura 5 - Correlações do primeiro (azul) e segundo (vermelho) fatores comuns temporais da componente meridional do vento à superfície em mesorregiões do estado da Bahia. (Fonte dos dados: Instituto do Meio Ambiente e Recursos Hídricos (INEMA))
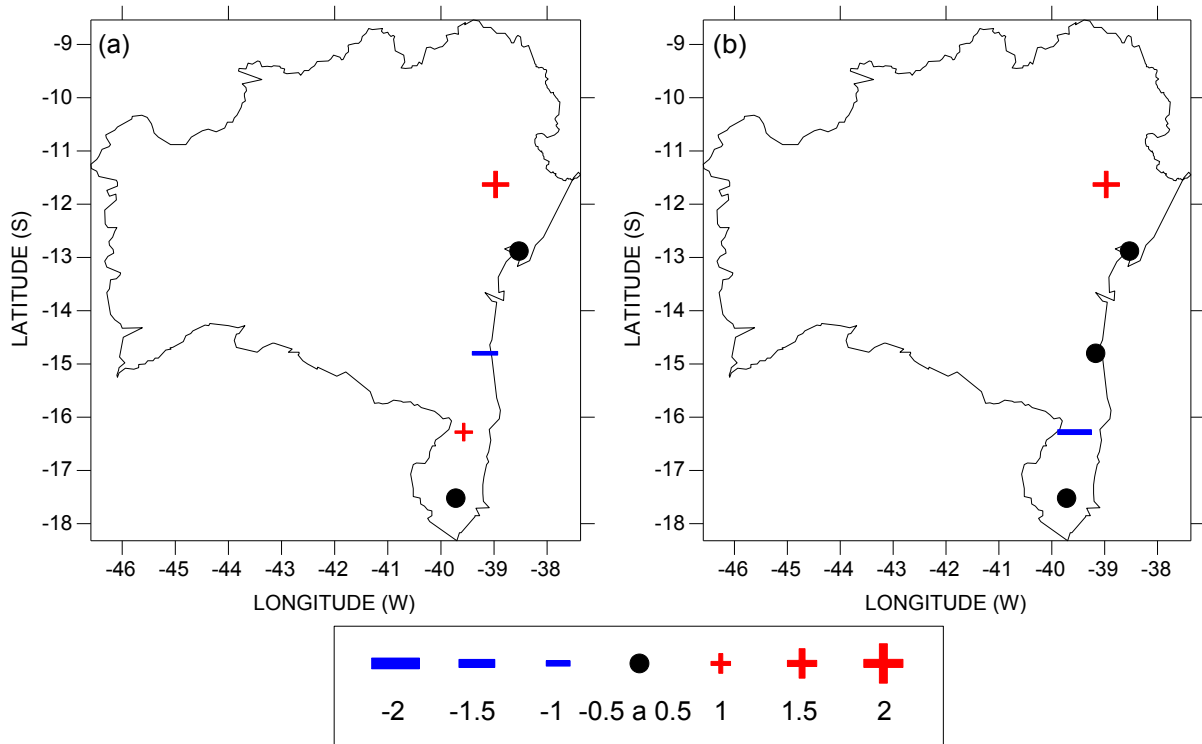

Figura 6 - Padrões de variabilidade espacial do primeiro (a) e segundo (b) fatores comuns temporais da componente meridional do vento à superfície em mesorregiões do estado da Bahia. As contribuições positivas estão representadas pelo símbolo (+) e as negativas pelo símbolo (-). (Fonte dos dados: Instituto do Meio Ambiente e Recursos Hídricos (INEMA))

primeira $\mathrm{CP}$ associada a este fator (Figura 6a) mostra contribuições positivas em Eunápolis e Serrinha, com valor máximo de 1,16 na última. As contribuições negativas são registradas em Salvador, Teixeira de Freitas e Ilhéus, com valor mínimo de $-1,34$ na última. Esse padrão de contribuições positivas e negativas indica que a componente meridional tem variabilidade semelhante no norte e no extremo sul do estado, em contraste com Ilhéus. Essa distribuição espacial indica que a interação entre o ASAS e os distúrbios de leste e sistemas frontais na área norte, e o ASAS e os sistemas frontais na área sul, é determinante na variabilidade do vento representada por este fator.

A segunda CP (Figura 6b) associada a este fator mostra contribuições positivas em Ilhéus, Teixeira de
Freitas e Serrinha, com valor máximo de 1,24 na última, e contribuições negativas em Salvador e Eunápolis, com valor mínimo de $-1,54$ na última. O contraste entre as áreas norte e sul do estado indicado por essa distribuição sugere a resposta local aos sistemas atmosféricos que atuam no trimestre NDJ, principalmente.

\subsection{Análise de agrupamento}

A partir dos dois fatores comuns temporais mais significativos da CZV, que explicaram 99,4\% da variância total, foi realizado o agrupamento das estações. Utilizando a curva de inércia (Figura 7a), foi feito um corte no dendrograma (Figura $7 \mathrm{~b}$ ), resultando em três (03) grupos homogêneos da CZV ilustrados na Figura 


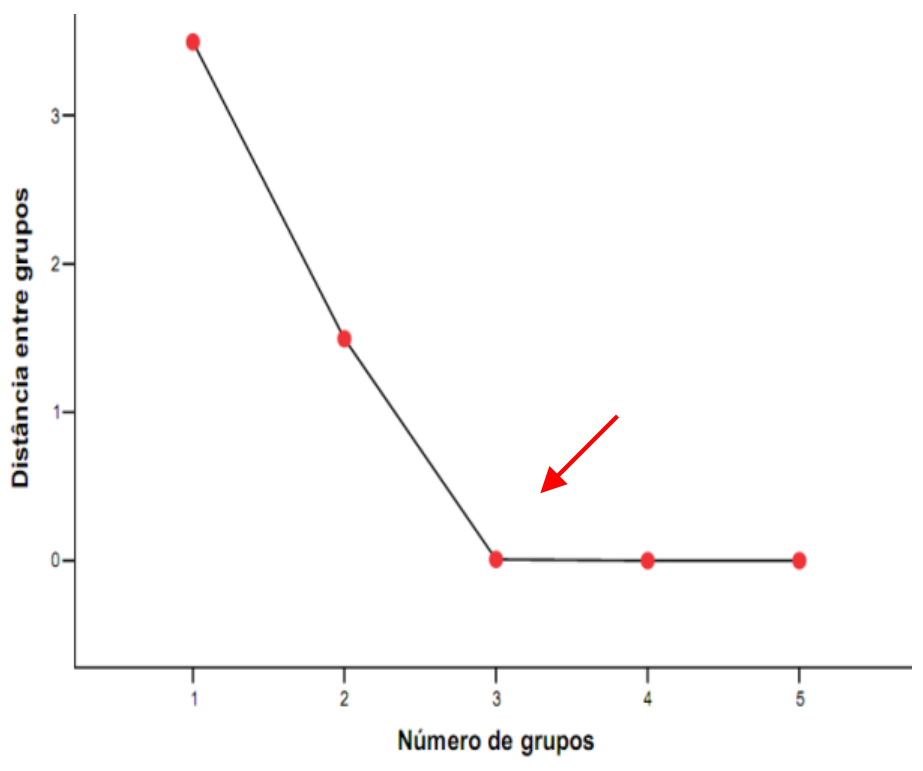

(a)

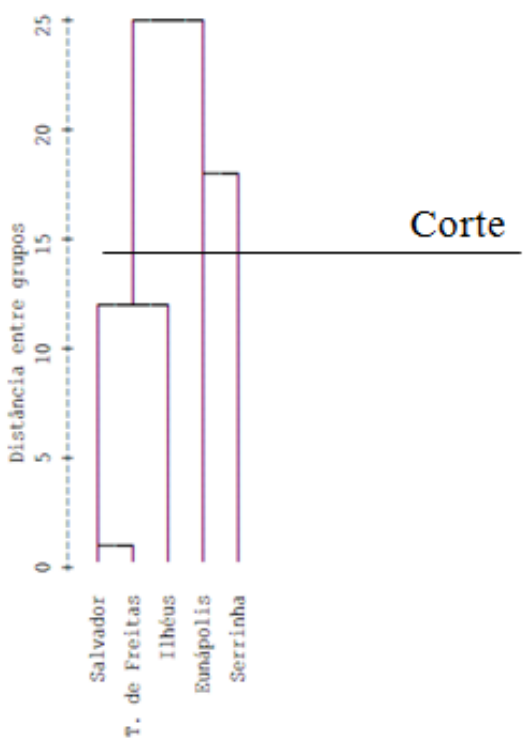

(b)

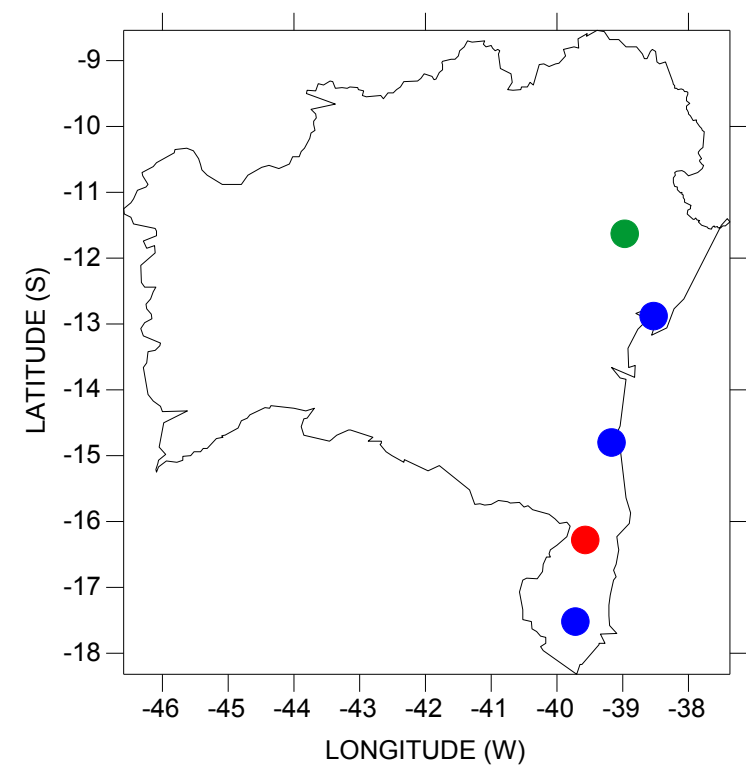

(c)

Figura 7 - Regionalização da componente zonal do vento à superfície: (a) curva de inércia com seta indicando o número de grupos obtidos, (b) dendrograma utilizando o método de Ward, e (c) zoneamento representado pelo primeiro (verde), segundo (azul) e terceiro (vermelho) grupos, realizado a partir dos dois primeiros fatores comuns temporais em mesorregiões da Bahia.

7c. As séries temporais dos valores médios trimestrais da CZV das estações que constituem cada grupo são mostradas na Figura 8. O Grupo I está constituído apenas por Serrinha (Figura 7c). A Figura 8a ilustra que a CZV é de leste nessa estação (valores negativos) e que é máxima no trimestre novembro-dezembro-janeiro (NDJ), com intensidade de $2,81 \mathrm{~ms}^{-1}$, e mínima no trimestre maio-junho-julho (MJJ), com $0,74 \mathrm{~ms}^{-1}$.
O Grupo II está constituído por Ilhéus, Salvador e Teixeira de Freitas (Figura 7c). A Figura 8b mostra a série temporal da CZV média desse grupo, mostrando valores negativos no período (componente de leste), com intensidades menores do que $1 \mathrm{~ms}^{-1}$. A CZV média é máxima no trimestre NDJ, com intensidade de $0,91 \mathrm{~ms}^{-}$ 1, e mínima no trimestre MJJ, com 0,13 $\mathrm{ms}^{-1}$. Este grupo exibe os menores valores da CZV, que é quase nula no 


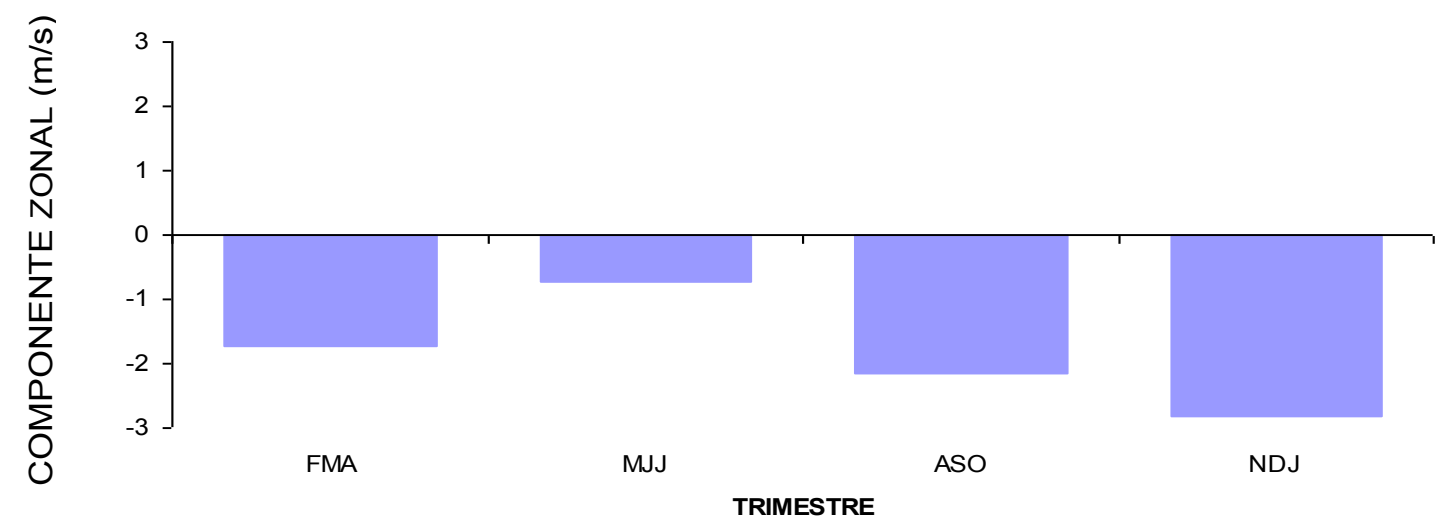

(a)
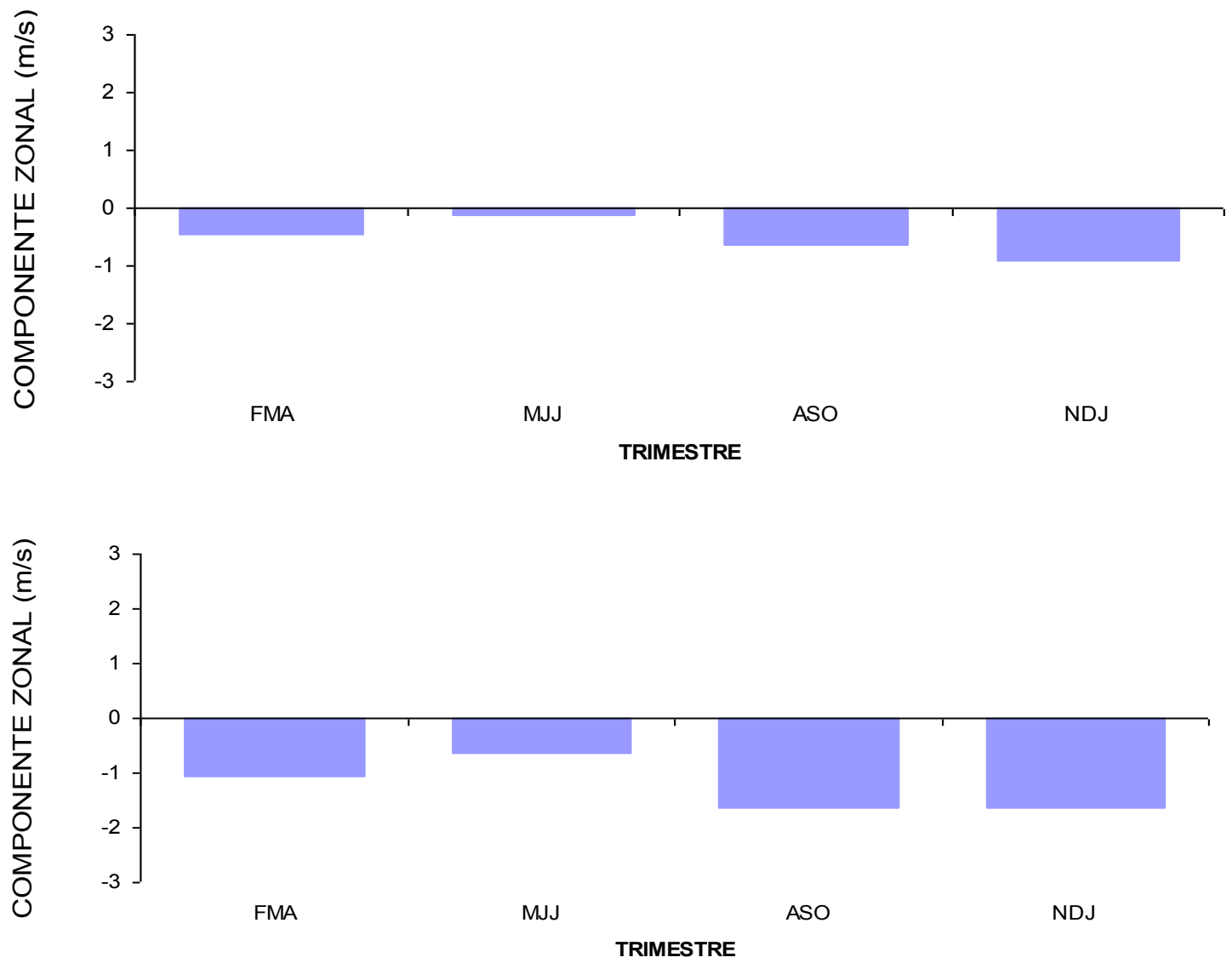

(c)

Figura 8 - Série temporal das médias trimestrais da componente zonal do vento à superfície (ms-1) do primeiro (a), segundo (b) e terceiro (c) grupos. Os valores negativos representam componente de leste. (Fonte dos dados: Instituto do Meio Ambiente e Recursos Hídricos (INEMA))

trimestre MJJ.

O Grupo III está constituído apenas por Eunápolis (Figura 7c). A Figura 8c ilustra a série temporal das médias trimestrais dessa estação, que apresenta comportamento semelhante ao dos grupos anteriores, com valores negativos (componente de leste). A CZV é máxima no trimestre NDJ, com valor de $1,65 \mathrm{~ms}^{-1}$, e mínima no trimestre MJJ, com valor de $0,64 \mathrm{~ms}^{-1}$.

A partir dos dois fatores comuns temporais mais sig- nificativos da CMV, que explicaram 99,6\% da variância total das médias trimestrais, foi feito o agrupamento das estações. Utilizando a curva de inércia (Figura 9a), foi feito um corte no dendrograma (Figura 9b), resultando em três (03) grupos homogêneos da CMV (Figura 10). As características dos grupos são descritas a seguir.

O Grupo I está constituído pela estação localizada em Serrinha (Figura 9c). A Figura 10a ilustra a série temporal das médias trimestrais da CMV dessa estação, exibindo 


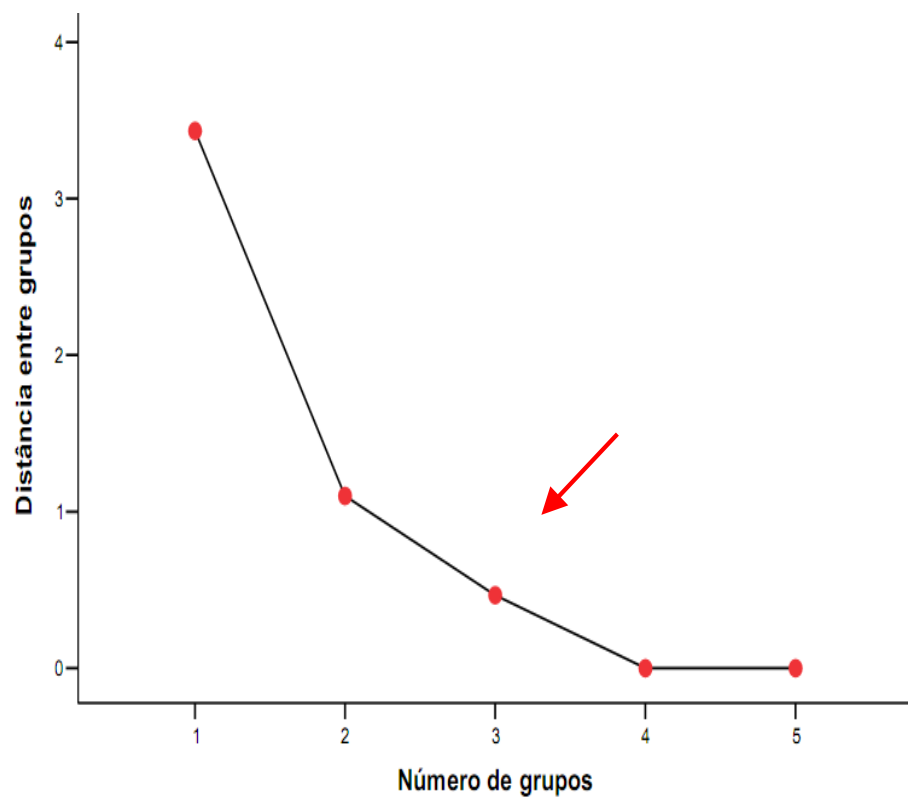

(a)
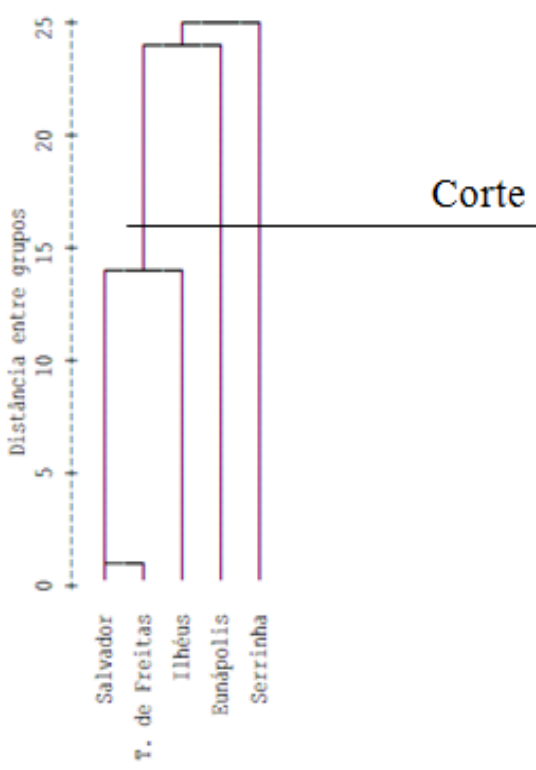

(b)

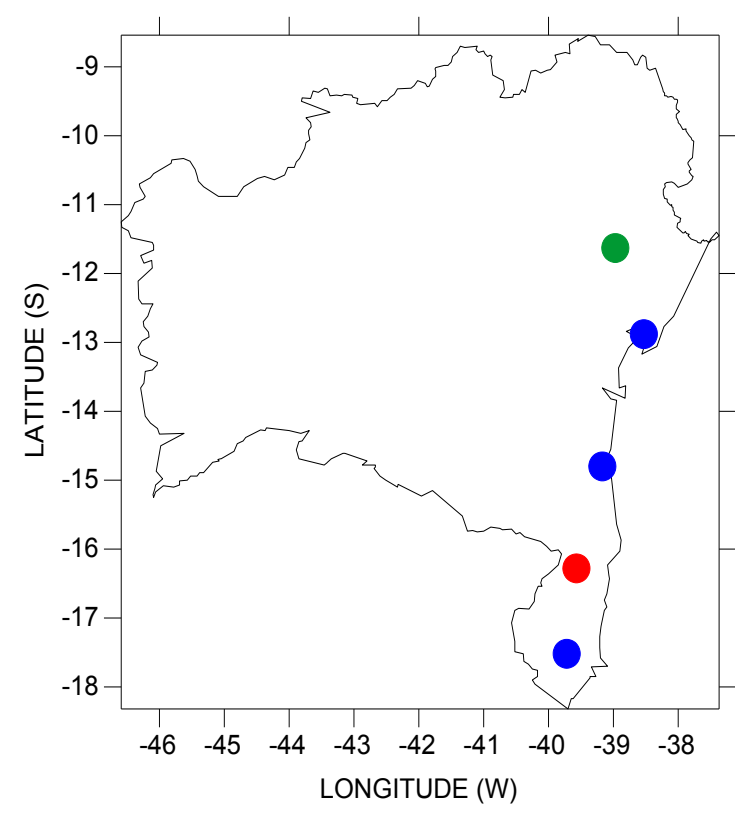

(c)

Figura 9 - Regionalização da componente meridional do vento à superfície: (a) curva de inércia com seta indicando o número de grupos obtidos, (b) dendrograma utilizando o método de Ward, e (c) zoneamento representado pelo primeiro (verde), segundo (azul) e terceiro (vermelho) grupos, realizado a partir dos dois primeiros fatores comuns temporais em mesorregiões da Bahia.

valores positivos, indicando que a componente é de sul. A CMV é máxima no trimestre MJJ, com valor de 1,85 $\mathrm{ms}^{-1}$, e mínima no trimestre NDJ, com valor de $0,1 \mathrm{~ms}^{-1}$. Este grupo apresenta os maiores valores da CMV no período estudado.

O Grupo II está constituído pelas estações localizadas em Ilhéus, Salvador e Teixeira de Freitas (Figura 9c). A Figura 10b ilustra a série temporal das médias trimestrais da CMV desse grupo; apenas o trimestre MJJ exibe valores positivos, demonstrando que a componente é de sul, enquanto que nos demais a componente é de norte. A intensidade da CMV é máxima no trimestre NDJ, com 


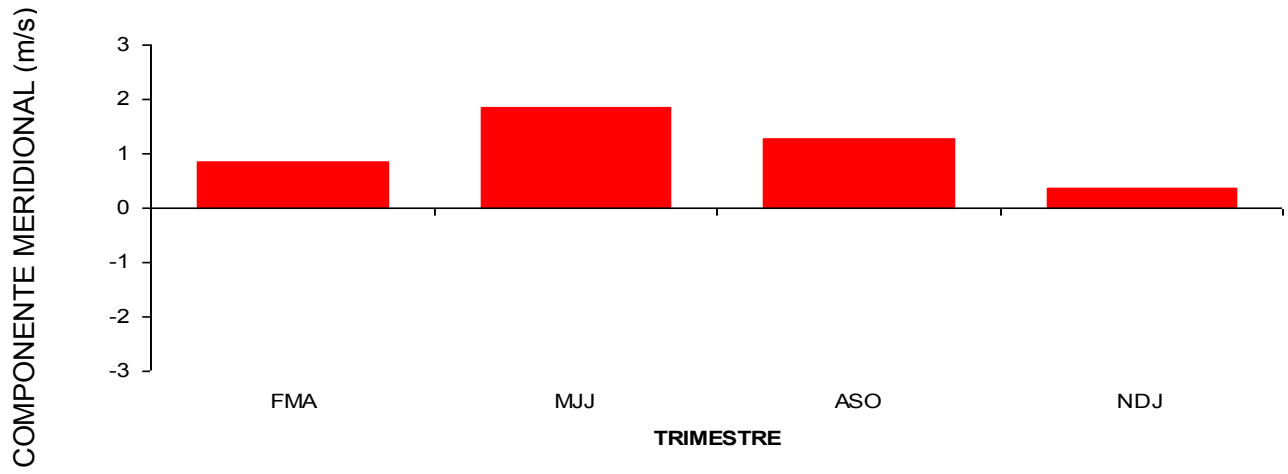

(a)

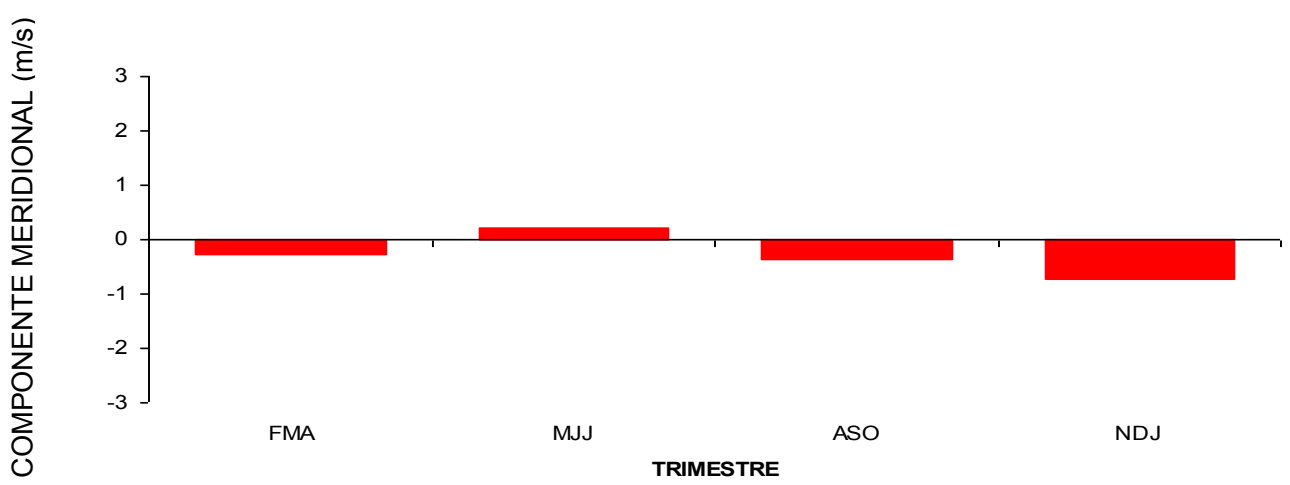

(b)

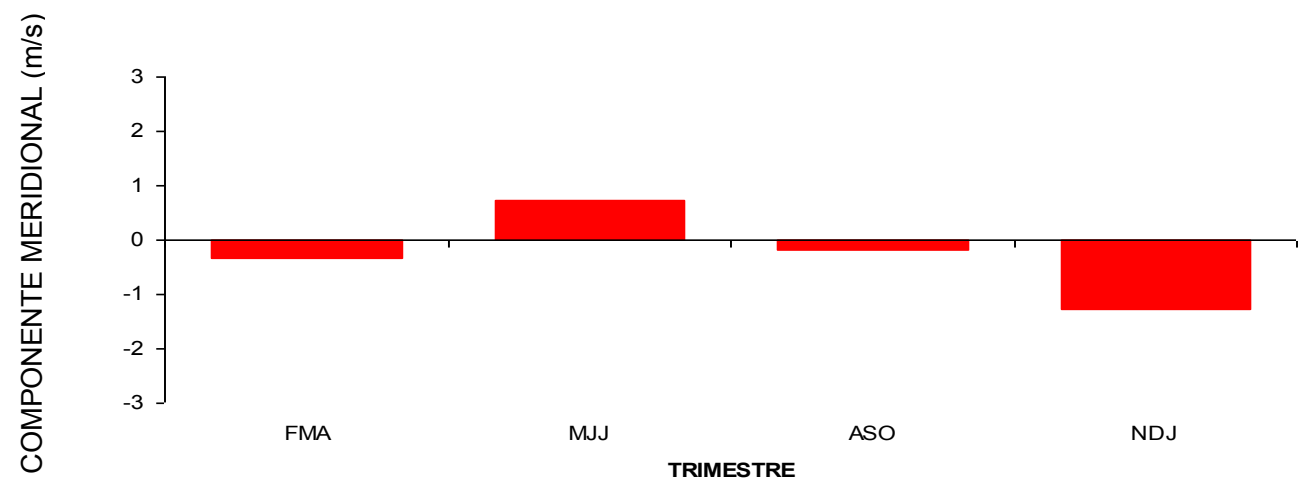

(c)

Figura 10 - Série temporal das médias trimestrais da componente meridional do vento à superfície $\left(\mathrm{ms}^{-1}\right)$ do primeiro (a), segundo (b) e terceiro (c) grupos. Os valores positivos (negativos) representam componente de sul (norte). (Fonte dos dados: Instituto do Meio Ambiente e Recursos Hídricos (INEMA))

valor de $-0,71 \mathrm{~ms}^{-1}$, e mínima no trimestre MJJ, com valor de $0,21 \mathrm{~ms}^{-1}$. Este grupo apresenta os menores valores da CMV no período estudado.

O Grupo III está constituído pela estação localizada em Eunápolis (Figura 9c). A Figura 10c ilustra a série temporal das médias trimestrais da CMV desse grupo. Semelhantemente ao Grupo II, apenas o trimestre MJJ exibe valores positivos, demonstrando que a componente é de sul, enquanto que nos demais a componente é de norte. A intensidade da CMV é máxima no trimestre $\mathrm{NDJ}$, com valor (negativo) de $-1,27 \mathrm{~ms}^{-1}$, e mínima no trimestre ASO, com valor (negativo) de $-0,19 \mathrm{~ms}^{-1}$.

\section{Conclusões}

Neste trabalho a variabilidade do vento à superfície em mesorregiões da Bahia foi investigada com o objetivo de identificar padrões temporais e regiões homogêneas usando técnicas de análise multivariada (análise de componentes principais e análise de agrupamento). Conclui-se que:

$\mathrm{Na}$ análise de componentes principais da componente zonal do vento (CZV) explicou 99,4\% da variância total das médias trimestrais a partir das duas primeiras $\mathrm{CP}^{\prime} \mathrm{s}$, com $96,1 \%$ e $3,3 \%$, respectivamente; 
Na análise de componentes principais da componente meridional do vento (CMV) explicou $99,6 \%$ da variância total das médias trimestrais a partir das duas primeiras $\mathrm{CP}^{\prime}$ 's, com $92,4 \%$ e $7,2 \%$, respectivamente;

A aplicação da análise de agrupamento aos fatores comuns temporais mais significativos através do método de Ward resultou em três grupos homogêneos, tanto para a CZV quanto para a CMV;

Verificou-se em Serrinha e Eunápolis que os sistemas atmosféricos interagiam com fatores locais como o relevo;

O anticiclone subtropical do Atlântico Sul (ASAS) e os sistemas frontais influenciaram na variabilidade no decorrer do período, e outros sistemas atmosféricos tais como: ZCAS, VCAS/VCAN e os distúrbios/ondas de leste afetaram em épocas específicas.

\section{Agradecimentos}

O primeiro autor agradece à Coordenadoria de Aperfeiçoamento de Pessoal de Ensino Superior (CAPES) pela concessão de Bolsa de Doutorado, ao Conselho Nacional de Desenvolvimento Científico e Tecnológico (CNPQ) pela concessão de Bolsa de Mestrado, e ao Instituto do Meio Ambiente e Recursos Hídricos (INEMA) pela cessão dos dados brutos das estações automáticas de coleta de dados. Este trabalho é parte da pesquisa do mestrado realizado no período de 2008-2010.

\section{Referências}

AIKAWA, M., HIRAKI, T, EIHO. Grouping and representativeness of monitoring stations based on wind speed and wind direction data in urban areas of Japan. Environmental Monitoring Assessment, v. 136, p. 411-418, 2008.

\section{ARCHER, C. L., JACOBSON, M. Z.}

Evaluation of global wind power. Journal of Geophysical Research, v. 110, p. 1-20, 2005, doi:10.1029/2004JD005462.

BARMPADIMOS, I., HUEGLIN, C., KELLER, J., HENNE, S., PRÉVÔT, S. H. Influence of meteorology on PM10 trends and variability in Switzerland from 1991 to 2008. Atmospheric Chemistry and Physics, v. 11, p. 1813-1835, 2011, doi:10.5194/acp-11-1813-2011.

BARRETO, A. B. Estudo do ciclo diário do vento à superfície no Nordeste do Brasil. 2001. 56 f. Dissertação (Mestrado em Meteorologia) Universidade Federal da Paraíba, Campina Grande, 2001.

BRAGA, C. C.; MELO, L. D. de; MELO, E. C. S.
Análise de agrupamento aplicada à distribuição da precipitação no Estado da Bahia. In: Congresso Brasileiro de Meteorologia, 10., Congresso da FLISMET, 8., 1998, Brasília. Anais... Rio de Janeiro: SBMet, 1998. CL-98001. 1 CD-ROM.

BURLANDO, M.; ANTONELLI, M.; RATTO, C. F. Mesoscale wind climate analysis: identification of anemological regions and wind regimes. International Journal of Climatology, v. 28, p. 629641, 2008.

CHAVES, R. R., CAVALCANTI, I. F. A. 2001:

Atmospheric Circulation features Associated with Rainfall Variability over Southern Northeast Brazil. Monthly Weather Review, v. 129, p. 2614-2626, 2001.

CORRAR, L. J; PAULO, E.; DIAS FILHO, J. M. Análise Multivariada - Para os Cursos de Administração, Ciências Contábeis e Economia, Ed. Atlas, São Paulo, 1를 Edição, 344 p., 2007.

CORREIA, A. A. Padrões de variabilidade do vento à superfície no Nordeste do Brasil. 2000. 66 f. Dissertação (Mestrado em Meteorologia) Universidade Federal da Paraíba, Campina Grande, 2000.

CORREIA FILHO, W. L. F. Variabilidade do Vento à Superfície em Mesorregiões da Bahia: Uma análise com Plataformas Automáticas de Coleta de Dados. 2010. 53 f. Dissertação (Mestrado em Meteorologia) - Universidade Federal de Campina Grande, Campina Grande, 2010.

EVERITT, B. Cluster Analysis. 3. ed. New York: Ed. John Wiley \& Sons Inc., 1993. 170p.

GONG, X.; RICHMAN, M. B. On Application of Cluster Analysis to Growing Season Precipitation Data in the North America East of the Rockies. Journal of Climate, v. 8, p. 897-931, 1995.

GREEN, M. C.; FLOCCHINI, R. G.; MYRUP, L. O. Use of Temporal Principal Components Analysis to Determine Seasonal Periods, Journal Applied Meteorology, v. 32, p. 986-995, 1993.

HUANG, W., TAN, J., KAN, H., ZHAO, N., SONG, W., SONG, G., CHEN, G., JIANG, L., JIANG, C., CHEN, R., CHEN, B. Visibility, air quality and daily mortality in Shanghai, China. The Science of the total environment, v. 407, p. 3295-3300, 2009, doi:10.1016/j.scitotenv.2009.02.019.

IBGE, Instituto Brasileiro de Geografia e Estatística. 
Mapa do relevo do Estado da Bahia. Disponível em: http://www.ibge.gov.br/ Acessado em Fevereiro de 2010.

JIMÉNEZ, P. A.; GONZÁLEZ-ROUCO, F.; MONTÁVEZ, J. P.; NAVARRO, J.; GARCÍAGUSTAMANTE, E.; VALERO, F. Surface Wind Regionalization in Complex Terrain. Journal of Applied Meteorology and Climatology, v. 47, p. 308-325, 2008.

KALKSTEIN, L. S., TAN, G., SKINDLOV, J. A. An evaluation of three clustering Procedures for Use in Synoptic Climatological Classification. Journal of Climate and Applied Meteorology, v. 26, p. 717730, 1987.

KAUFMANN, P.; WHITEMAN C. D. ClusterAnalysis Classification of Wintertime Wind Patterns in the Grand Canyon Region. Journal of Applied Meteorology, v. 38, p.1131-1147, 1999.

KASSOMENOS, P. A.; SINDOSI, O. A.; LOLIS, C. J. Seasonal variation of the circulation types occurring over southern Greece: a 50 yr study. Climate Research, v. 24, p. 33-46, 2003.

KLINK, K. Climatological means and interannual variance of United States surface wind speed, direction and velocity. International Journal Climatology, v. 19, p. 471-488, 1999.

KOUSKY, V. E. Frontal Influences on Northeast Brazil. Monthly Weather Review, v. 107, p. 11401153, 1979.

MOLION, L.C.B, BERNARDO, S.O. Uma Revisão da Dinâmica das Chuvas no Nordeste Brasileiro. Revista Brasileira de Meteorologia, v.17, n. 1, p. 1-10, 2002.

MOSCATI, M. C. de L.; GAN, M. A. Rainfall variability in the rainy season of semiarid zone of Northeast Brazil (NEB) and its relation to wind regime. International Journal Climatology, v.27, p. 493-512, 2007.

OLIVEIRA SILVA, P. K. ; SILVA ARAGÃO, M. R.; CORREIA, M. F. Rajadas de vento na região metropolitana de Salvador, Bahia. Ciência e Natura, v. 10, p. 185-188, 2009.

OLIVEIRA SILVA, P. K. ; SILVA ARAGÃO, M. R.; CORREIA, M. F.; BARBOSA SILVA, A.; SANTOS, S. A. Detecção de padrões homogêneos de vento. Parte 2: Análise de agrupamento. Ciência e Natura, v. 12, p. 347-349, 2011.
QIAN, Z., HE, Q., LIN, H.-M., KONG, L., LIAO, D., DAN, J., BENTLEY, C. M., WANG, B. Association of daily cause-specific mortality with ambient particle air pollution in Wuhan, China. Environmental Research, v. 105, p. 380-389, 2007, doi:10.1016/j.envres.2007.05.007.

RAO, V. B.; LIMA, M. C. de; FRANCHITO, S.H. Seasonal and Interannual Variations of Rainfall over Eastern Brazil. Journal of Climate, v. 6, p. 1754-1763, 1963.

SANTOS, A. H. M. Eventos extremos de chuva em Salvador, Bahia: Condições atmosféricas e impactos ambientais. 2008. 65 f. Dissertação (Mestrado em Meteorologia) - Universidade Federal de Campina Grande, Campina Grande, 2008.

SANTOS, A. H. M.; SILVA ARAGÃO, M. R.; CORREIA, M. F.; ARAÚJO, H. A.; BARBOSA SILVA, A. Distúrbio ondulatório de leste e seus impactos na cidade de Salvador. Revista Brasileira de Meteorologia, v. 27, n. 3, p. 355-364, 2012.

SILVA, B. B. da; ALVES, J. J. A.; CAVALCANTI, E. P.; DANTAS, R. T. Potencial eólico da direção predominante do vento no Nordeste do Brasil. Revista Brasileira de Engenharia Agrícola e Ambiental, v.6, nº 3, p. 431-439, 2002.

WARD, J. H. Hierarchical grouping to optimize an objective function. Journal American Association, v. 58, p. 236-244, 1963.

WEBER, R. O.; FURGER, M. Climatology of near-surface wind patterns over Switzerland. International Journal Climatology, v. 21, p. 809-827, 2001.

WILKS, D. Statistical Methods in the Atmospheric Sciences, Ed. Academic Press, London, 592p., 2005. 